\title{
HACIA LA CONSTRUCCIÓN DE LAS PRÁCTICAS COMUNITARIAS DE TERAPEUTAS OCUPACIONALES EN CHILE, DESDE UNA MIRADA SOCIO HISTÓRICA, DESDE 1972 HASTA LA ACTUALIDAD
}

\section{Towards the construction of the Occupational Therapists community practices in Chile, from a social historic view, from 1972 until nowadays}

\author{
Oyarzun S, Nataly; ${ }^{\text {i }}$ Zolezzi G., Roxanna ${ }^{\text {ii. }}$ Núñez S., Juan ${ }^{\text {iii. }}$ Palacios T., Mónica ${ }^{\text {iv }}$.
}

\section{RESUMEN}

Esta investigación, muestra la construcción de prácticas de Terapeutas Ocupacionales que, desde diversas perspectivas, han realizado trabajo comunitario en Chile, pretendiendo comprender su desarrollo en distintos contextos históricos desde 1972 hasta la actualidad, rescatando relatos en base a la experiencia, que den cuenta de la relación entre contextos socio históricos y prácticas comunitarias.

La metodología utilizada para este estudio fue cualitativa. Se recopiló información mediante once entrevistas semiestructuradas y un grupo de discusión. Posteriormente, se analizó la información a través de Teoría Fundada, en diferentes categorías, abierta y axial con un eje central en discusión con planteamientos teóricos.

Los hallazgos de este estudio, permiten concluir que los contextos socio históricos determinan las formas de hacer prácticas comunitarias en Chile; y éstas, a su vez, influyen en la interacción con la comunidad favoreciendo o dificultando su participación social. El Terapeuta Ocupacional es parte de ese contexto sociohistórico por lo que su identidad, equipo de trabajo y su práctica comunitaria está configurada y construida desde ahí, sin embargo, el no hacer consciente sus prácticas y cómo éstas se han construido, no permitiría establecer la dimensión política de su hacer. Ocuparse de la ocupación, junto con la cotidianeidad en el campo comunitario implica una comprensión de la realidad socio-histórica, determinando las prácticas comunitarias de los Terapeutas Ocupacionales así como la construcción de comunidad.

Palabras Claves: Contexto Sociohistórico, Comunidad, Practica Comunitaria, Intervención comunitaria, Trabajo Comunitario, Cotidianidad, Participación

\footnotetext{
i T.O. Licenciada en Ciencias de la Ocupación, Universidad Mayor. Fono 9 3046742, Email: nataly.oyarzun@gmail.com

ii T.O. Licenciada en Ciencias de la Ocupación, Universidad Mayor. Fono 8 4096430, Email: roxzole@hotmail.com

iii Trabajador Social, Licenciado en Trabajo Social, Universidad Arcis, Docente Universidad Mayor. Fono 95826282, E-mail: juannunezs@gmail.com

iv T.O. Candidata Magíster Psicología social Comunitaria, Universidad Católica, Diplomada Salud Familiar, Universidad de Chile, Docente Universidad Mayor. Fono 9 7896537, E-mail: monica.palacios@umayor.cl
} 


\section{ABSTRACT}

This investigation will show the process of constructing Occupational Therapy practices that have been realized from various perspectives via community work in Chile, attempting to understand its development in the different social and historical periods since 1972 until the present time, emphasizing experience accounts, wich make clear the relation between social and historical contexts and practices.

The methodology employed in this work was qualitative with information based on eleven semi-structured interviews and a discussion group. The information was analyzed using the Grounded Theory, through opened and axial categories, related to a central theoretical axis.

The findings conclude that the social and historical contexts determine the way of doing community practice in Chile, and these affect the interaction with community, favoring or making difficult their social participation. The therapist is part of that social and historical context. Therefore, his identity, work team and community practice are constructed there. Nevertheless, if he does not become conscious of his practice and how it has been constructed, he will not build the political dimension of his work. To be in charge of daily occupation in the community field involves an understanding of the social and historical reality, determining the community practices of the Occupational Therapists and likewise the construction of community.

Key words: socio historical context, community practices, community intervention, community work, participation, everyday

\section{INTRODUCCION}

En la siguiente investigación se mostrarán distintas experiencias de un grupo de Terapeutas Ocupacionales que han desarrollado prácticas comunitarias en diferentes momentos históricos en Chile, específicamente desde el año 1972 hasta la actualidad. De este modo, se reconstruirán las experiencias de Terapeutas Ocupacionales de diversas generaciones, para comprender como se ha desarrollado el trabajo comunitario en la profesión.

Para situarnos en la práctica comunitaria es necesario revisar la noción de comunidad. Autores como Maritza Montero plantean que los procesos de participación social y de fortalecimiento generan un rol activo de las comunidades, siendo éstas un soporte social efectivo para diversas problemáticas. Sin embargo, en la actualidad se evidencia una tendencia orientada hacia la individualización de las personas y una escasa participación social; la pérdida de la confianza en el vecino, la falta de soporte del tejido social y un sinnúmero de emergentes psicosociales, hacen imprescindible volver a trabajar para potenciar el rol de las comunidades ${ }^{1}$

Es en relación a lo anterior que los Terapeutas Ocupacionales han desarrollado diversas experiencias con comunidades como sujetos de intervención. Para comprender como la Terapia Ocupacional se ha vinculado a lo comunitario, es necesario entender su historia y evolución como disciplina desde sus comienzos hasta su actual desarrollo en Chile, sitúada en distintos momentos históricos, políticos y sociales el país. 
El interés de este estudio, es abarcar los aspectos relacionados con la historia de la construcción de las prácticas comunitarias de la Terapia Ocupacional, cómo han sido ejercidas, cómo se han encontrado con la teoría y cómo han sido las experiencias de Terapeutas Ocupacionales que han aportado ha este campo.

\section{FUNDAMENTACION}

En la información recolectada por las autoras de este estudio, se evidencia que existen escasos registros sistematizados de prácticas de Terapia Ocupacional en la comunidad. Esto, ha hecho que los desarrollos y sustentos teóricos que fundamentan el quehacer comunitario, se hayan desarrollado principalmente desde otras disciplinas.

Se parte del supuesto de las autoras que el trabajo comunitario desarrollado por Terapeutas Ocupacionales, comenzó en base a experiencias particulares de éstos y no desde aspectos teorícos específicos, es decir, desde una historia de vida e interés personal que facilitó su vinculación con este campo, determinado a su vez por el contexto socio histórico, en donde el acercamiento a una comunidad, incide en la formacióndel Terapeuta Ocupacional. Por lo tanto, las autoras consideran que estos han tenido que desarrollar estrategias para generar cambios no sólo desde las comunidades, sino también desde sí mismos.

A nivel nacional, existe escasa evidencia de trabajo comunitario, publicada en la literatura de Terapia Ocupacional. Es en este ámbito donde radica la importancia del presente estudio y sus posibles aportes hacia el desarrollo de las prácticas de Terapeutas Ocupacionales en la comunidad.

Al revisar los antecedentes históricos de la Terapia Ocupacional, encontramos que en el siglo XVI, Pinel, Psiquiatra Francés ${ }^{2}$, plantea un tratamiento caracterizado por la reducción del espacio asilar y la actividad como herramienta terapéutica, donde la realización de actividades con sentido real y provisto de utilidad material y social, facilitan la reinserción de la persona en su entorno comunitario y social. Lo anterior, en conjunto con el movimiento de artes y oficios (1860), constituye algunas de las raíces de la Terapia Ocupacional, y a través de ellos puede apreciarse de manera incipiente aspectos relacionados a los contextos y la comunidad. Al llegar la primera (1914-1918) y segunda (1939-1945) guerras mundiales, los aspectos revisados pierden dominancia ya que por efectos de la guerra, fue necesario acelerar la recuperación de las personas de manera rápida y eficiente para devolver a la sociedad personas productivas, dando énfasis a la rehabilitación física desde espacios hospitalarios.

En Chile, la Terapia Ocupacional, tiene sus raíces en 1963 en la Universidad de Chile, en el área de la psiquiatría y la salud física. En el comienzo de la carrera, el énfasis de la formación era desde un enfoque médico tradicional $\mathrm{y}$ en el aprendizaje de técnicas grupales y ergoterapias.

En 1971 surge el movimiento de la reforma universitaria por acción de los propios estudiantes y se realiza la primera convención de Terapia Ocupacional. Con esto, se logra la modificación del plan de estudios, incluyendo asignaturas de formación general y las asignaturas de formación clínica y profesional. En este periodo 70-73 (que para efectos del presente estudio llamaremos Pre-dictadura) el enfoque dominante en la formación académica sigue siendo biomédico. Sin embargo en el país y en algunas prácticas de salud se generan acciones en las poblaciones y comunidades, como las promovidas por los psiquiatras L. Weinstein y J. Marconi el primero con la salud poblacional y el segundo con la psiquiatría comunitaria. De 
manera informal, a través de los trabajos sociales realizados por los jóvenes universitarios desde posicionamientos políticos y partidarios, se generaron escenarios que permiten la participación de algunos terapeutas ocupacionales desde una formación ligadas a experiencias paralelas a lo académico, como: la participación en grupos sociales, militancia política, etc. En 1973, al instalarse en el poder la dictadura militar, los convenios entre el Servicio Nacional de Salud y la Universidad de Chile se rompen, y muchos Terapeutas Ocupacionales, son cesados en sus funciones. Esto provoca que los Terapeutas Ocupacionales, abrieran nuevos espacios de acción en el ámbito profesional y tuvieran que desarrollarse en diversos contextos, en los que no tenían una experiencia previa. Durante el periodo de 1973 hasta 1989, que comprende la dictadura, la formación académica estaba definida desde las áreas de salud física y salud mental, en donde no existía cabida formal para el trabajo poblacional desarrollado en esa época ${ }^{3}$.

En 1994, se incorpora a la malla de la Universidad de Chile, el ramo de Terapia Ocupacional Comunitaria y, con posterioridad, en las Universidades de Playa Ancha, Andrés Bello, Mayor, Austral y Universidad Autónoma. Actualmente, en las escuelas de Terapia Ocupacional, tanto estatales como privadas, se integra la asignatura comunitaria, en su mayoría desde cuarto año, con diferentes énfasis según el lineamiento de cada Universidad y Escuela. Lo relevante de este aspecto, es que se ha instalado lo comunitario en la formación de pregrado, con lo que las nuevas generaciones de Terapeutas Ocupacionales tienen un desarrollo teórico con respecto al trabajo comunitario. Es relevante mencionar que, si bien se ha incorporado en las mallas curriculares, no se ha investigado ni sistematizado acerca de la historia de este ámbito de la profesión.

En la búsqueda de documentos que vinculen la Terapia Ocupacional con la comunidad, se encuentran sistematizaciones de prácticas en Estados Unidos y Europa, éstas separan los aspectos individuales de los comunitarios como distintas intervenciones, en donde lo comunitario se asocia a un campo de intervención que funciona como medio para integrar a las personas a la comunidad. ${ }^{4}$

En el libro "Terapia Ocupacional sin Fronteras" 5 (nota al pie), se describen las experiencias de Terapeutas Ocupacionales y de otras profesionales afines que han puesto en marcha prácticas comunitarias en distintos países, haciendo aportes teóricos en relación a la Terapia Ocupacional, estableciendo distintos conceptos como el de justicia ocupacional y el de apartheid ocupacional ${ }^{\mathrm{v}}$, en los que se desafía a los Terapeutas Ocupacionales a desarrollar la visión social de la profesión.

En el artículo “Ocupación y significado: Aportes de Terapia Ocupacional desde un modelo constructivista”, 6 la autora, aporta a la Terapia Ocupacional desde la psicología, respecto a la problematización de los aspectos teóricos que son llevados a las prácticas de los Terapeutas Ocupacionales. Invita a reflexionar acerca del entramado constructivista y sus posibles aportes a Terapia Ocupacional, mencionando que implementar actividades con propósito no es suficiente, si no se explora el modo particular de significar a las personas, instituciones y comunidades, desde problemáticas como las relaciones de poder, los condicionantes raciales, socioeconómicos, de género, la desocupación, la violencia, las adicciones y la sexualidad, si se quieren lograr cambios sostenidos y enriquecedores.

\footnotetext{
${ }^{v}$ Apartheid ocupacional. se refiere a la separación social de los que no tienen acceso a ocupaciones significativas y útiles, aquellos que están privados de tales ocupaciones, o están limitadas en su vida diaria.

Justicia Ocupacional: hace referencia a favorecer la oportunidad y recursos requeridos para la participacion ocupacional que permita satisfacer las necesidades personales y ciudadania plena. (5)
} 
En Chile, existen dos artículos, publicados en la revista de Terapia Ocupacional de la Universidad de Chile, El primer llamado, "La Terapia Ocupacional en la intervención en salud comunitaria: el modelo educativo" y el segundo "Intuiciones del hacer, reflexiones acerca del quehacer en la intervención comunitaria” ${ }^{8}$. Ambos artículos, plantean la relación de la terapia ocupacional con el trabajo comunitario desde la salud y la atención primaria, mostrando una experiencia acerca del trabajo comunitario y el rol del Terapeuta Ocupacional. En ellos se señala que no es suficiente reproducir el trabajo institucional en ambientes comunitarios, sino que para trabajar en el marco de promoción de la salud, es necesario, un cambio de paradigma. Se hace una reflexión acerca de lo comunitario como un área del hacer del Terapeuta Ocupacional, y las dificultades en el actuar desde la atención primaria en salud.

Con respecto a la sistematización del trabajo comunitario, merece una mención especial, la ONG Educación Popular en Salud (EPES), que nació en Santiago, como parte de la acción diacónica de la Iglesia Evangélica Luterana en Chile (IELCH) en 1982, con el fin de fomentar la organización y participación comunitaria en salud de sectores pobres, basados en educación popular y la participación de las comunidades en las estrategias para la resolución de sus problemas. En esta experiencia han participado activamente Terapeutas Ocupacionales en el trabajo de promoción de salud, colaborando en artículos y documentos de experiencias comunitarias en Chile sistematizados por la ONG EPES. ${ }^{9}$

En base a lo señalado, surge la inquietud de conocer como los contextos socio-históricos, desde 1972 hasta la actualidad, influyen en las motivaciones, significados, fundamentos teóricos y experiencias; que hicieron posible realizar prácticas comunitarias. Así mismo, promover el conocimiento de éstas en las siguientes generaciones de Terapeutas Ocupacionales.

\section{METODOLOGÍA DE LA INVESTIGACIÓN}

La metodología de esta investigación, es de carácter cualitativa, se estudia el fenómeno en cuestión desde los relatos de quienes han sido parte del campo de estudio. La metodología cualitativa, permite profundizar en aspectos que para efectos de esta investigación son relevantes como son las experiencias y los significados para los Terapeutas Ocupacionales que realizaron prácticas comunitarias (1).

Se optó por un diseño de carácter Exploratorio - Descriptivo. Exploratorio debido a que, según los antecedentes encontrados, es un campo que ha sido poco estudiado en Chile y Descriptivo, porque da cuenta de un fenómeno determinado y se caracteriza a partir de los relatos de sus protagonistas a la luz de los distintos períodos históricos en los cuales se ha desarrollado la experiencia. Con esto, se pretende dar un primer paso, para que en un futuro se puedan impulsar otras investigaciones y profundizar a partir de este tema.

La selección se realizó en base a tres criterios, el primero, que hayan realizado prácticas comunitarias en su ejercicio profesional, el segundo, que su generación de egreso de la carrera de Terapia Ocupacion, correspondiera a alguno de los tres períodos históricos en los cuales se divide el estudio. el tercer criterio, es la aceptación voluntaria, por medio de consentimiento informado, a participar del proceso de investigación. Finalmente fueron seleccionados once Terapeutas Ocupacionales. 
Cuadro Resumen Selección de Participantes

\begin{tabular}{|c|c|c|c|c|}
\hline Entrevistados1 & Edad & Año de Egreso & Universidad & Prácticas comunitarias \\
\hline Entrevistado 1 & 58 & 1972 & De Chile & $\begin{array}{l}\text { Experiencias comunitarias en Nicaragua, } \\
\text { trabajo con mujeres en Lota y en la } \\
\text { Comunidad Terapéutica de Peñalolén }\end{array}$ \\
\hline Entrevistado 2 & 52 & 1977 & De Chile & $\begin{array}{l}\text { Trabajo voluntario en la cárcel, ONG } \\
\text { Forjamundos y trabajo en gendarmería }\end{array}$ \\
\hline Entrevistado 3 & 44 & 1987 & De Chile & $\begin{array}{l}\text { Trabajo comunitario en población Jorge } \\
\text { Hirmas, ITAK, trabajo en ONG de Renca, } \\
\text { proyecto PDJ de FOSIS en Huechuraba } \\
\text { (La Pincoya), ONG "La esquina" en La } \\
\text { Pintana, } \\
\text { ONG ECO en educación popular, los } \\
\text { Navíos, Corporación Chasqui. } \\
\end{array}$ \\
\hline Entrevistado 4 & 43 & 1987 & De Chile & $\begin{array}{l}\text { ONG EPES, COSAM de Independencia, } \\
\text { proyectos comunitarios en distintas } \\
\text { comunas y desde el Estado en FONADIS }\end{array}$ \\
\hline Entrevistado 5 & 47 & 1985 & De Chile & $\begin{array}{l}\text { Proyecto "Familia Solidaria” La Florida, } \\
\text { Asociación chilena de prevención del Sida } \\
\text { y desde el Estado en MIDEPLAN, FOSIS } \\
\text { y FONADIS }\end{array}$ \\
\hline Entrevistado 6 & 32 & 1998 & De Chile & $\begin{array}{l}\text { Práctica en ONG EPES y en consultorio } \\
\text { Orlando Letelier (APS) }\end{array}$ \\
\hline Entrevistado 7 & 32 & 1997 & De Chile & $\begin{array}{lll}\text { ONG Reencuentros, COSAM PAC, } \\
\text { CETRAM }\end{array}$ \\
\hline Entrevistado 8 & 34 & 1995 & De Chile & $\begin{array}{lll}\text { ONG CINTRAS, COSAM de } \\
\text { Huechuraba }\end{array}$ \\
\hline Entrevistado 9 & 33 & 1995 & De Chile & $\begin{array}{l}\text { Práctica profesional en el EPES, Proyecto } \\
\text { en servicio de salud - oriente "desarrollo } \\
\text { integral del niño", centro comunitario } \\
\text { Jorgito Fabio en Algarrobo, Trabajo en } \\
\text { proyectos en Ñuñoa y San Joaquín }\end{array}$ \\
\hline Entrevistado 10 & 30 & 2006 & Mayor & $\begin{array}{l}\text { Voluntariado con niños con Síndrome de } \\
\text { Down, COSAM El Bosque }\end{array}$ \\
\hline Entrevistado 11 & 32 & 2002 & De Chile & $\begin{array}{l}\text { Proyectos comunitarios de RBC, ONG } \\
\text { SEDEJ la Victoria, El Monte y La } \\
\text { Bandera }\end{array}$ \\
\hline
\end{tabular}

Las técnicas de selección fueron, “informantes claves” y "Bola de nieve”. La primera permite contar con sujetos seleccionados por el conocimiento, experiencia y vínculo que tienen con el fenómeno que se investiga. Es decir Terapeutas Ocupacionales que han realizado prácticas de carácter comunitario. La segunda técnica permite que sean los propios participantes los que recomiendan y establecen los contactos con otros Terapeutas Ocupacionales de similares características.

\section{Recopilación de Información}

La primera técnica de recopilación fue la entrevista semi estructurada, lo esencial es que el entrevistador guía la entrevista y es el entrevistado quien tiene la palabra, los entrevistados se puedan extender y profundizar en las temáticas, pero sin desviarse del problema de investigación. Se diseñó una guía con 19 preguntas ordenadas en ejes temáticos vinculados a: historia personal, antecedentes académicos, motivaciones, contextos, aspectos teóricos, concepto de comunidad, intervenciones, proyecciones y aspectos generales de lo comunitario.

Además, se realizó un grupo de discusión, en el que participaron cinco de los Terapeutas Ocupacionales entrevistados. La realización del grupo de discusión permitió triangular la información obtenida en las entrevistas individuales con el discurso colectivo construido por 
los participantes. Al grupo de discusión asistieron: un Terapeuta Ocupacional de la generación de los 77, otro de los 87 (periodo de dictadura) y tres de las generación de los 90(periodo post dictadura), se excusó de asistir la persona correspondiente al periodo predictadura. Esta instancia contó con una guía temática de dos puntos: aspectos formativos y prácticas comunitarias, que emergieron de las entrevistas individuales.

\section{Análisis de la Información}

El análisis de la información se realizó en base a la Grounded theory o Teoría Fundada ${ }^{10}$, se realizó una primera categorización abierta respecto de los conceptos claves que aparecieron desde de los discursos de los participantes, esta relación derivó en un nuevo proceso de categorización axial que permitió establecer relaciones tanto para los elementos teóricos preestablecidos, como para aquellos emergentes.

De este proceso resultaron tres categorías de análisis: Aspectos formativos; Motivaciones de los Terapeutas Ocupacionales comunitarios; Práctica de la Terapia Ocupacional Comunitaria. Estas categorías fueron analizadas desde un eje transversal a todas ellas: Contexto Sociohistórico

Para facilitar el análisis de las entrevistas personales, se realizaron cuadros por cada entevista individual y posteriormente por cada categoría. Además se realizaron matrices de análisis comparativas grupales, en donde el primero estaba formado por los Terapeutas Ocupacionales, de la generación de los 70 y 80 y otro de las generaciones de los 90 y 2000. Sumado a esto, se realizaron mapas conceptuales, como recurso metodológico, para ordenar y cruzar la información con respecto al análisis. Con ese proceso, se desarrollaron los resultados y el análisis presentado en el siguiente punto.

\section{RESULTADOS}

A continuación, se muestran los testimonios que, según los Terapeutas Ocupacionales entrevistados, construyen la historia de las prácticas comunitarias en Chile, ordenados en el eje de análisis transversal y las tres grandes categorías ya señaladas.

El contexto socio histórico previo a la dictadura se asocia a un período donde la sociedad y lo comunitario se concebían como un camino que favorecía la participación ${ }^{11}$. Existía sensibilidad política frente a las desigualdades, y la sociedad civil quería apropiarse de su historia, y sus derechos. El trabajo poblacional estaba orientado al fortalecimiento de la unidad popular y el gobierno. El agente de cambio es el sujeto colectivo.

“...se estaba iniciando entonces, que además tenía que ver con todo el mundo, donde se había iniciado todo un proceso en que era importante trabajar con la gente, y la gente participara en su salud, para prevenir, incluso para curar si es que se trabajara afuera en las poblaciones con la gente..." (Entrevistado 1, generación del 72)

El segundo período histórico revisado en esta investigación corresponde al período de la dictadura, el cual genera un cambio en el desarrollo de las prácticas comunitarias en el país. El trabajo en las poblaciones se volvió clandestino, ligado a la iglesia y a las ONG, las cuales eran financiadas por organismos internacionales. Por otra parte existía una organización comunitaria que se potenciaba, en base a una problemática común dada por la dictadura.

“...La consigna era de Trabajo popular y de crear poder popular...”.... "En los 80 hubo un protagonismo de tomar las riendas de la historia y poder generar un cambio histórico. Aportamos para que ese cambio histórico sucediera, más allá de que los resultados no fueran los que yo quería, pero hubo un cambio...”. (Entrevistado 3, generación del 87) 
Al llegar el tercer período histórico (post dictadura), el Estado se hace cargo de lo comunitario a través de las políticas públicas, las ONG pierden financiamiento internacional y en su mayoría desaparecen. Las que sobreviven lo hacen financiando sus actividades a través de proyectos gubernamentales, el Estado, define las prestaciones para las diversas comunidades, es decir, el trabajo comunitario se hace desde las instituciones y se instrumentaliza. Las comunidades pierden protagonismo y el trabajo vuelve a centrarse en lo individual.

“...Estamos en un modelo neoliberal de economía, centrado en lo individualismo, centrado en el consumismo, eso creo que no hay oportunidad para lo distinto, se busca la norma, no hay oportunidades, aparece la frustración, porque siempre hay topes la posibilidad de cambio cada vez se torna más difícil...” (Entrevista 10, generación del 2006)

\section{Aspectos Formativos.}

Para efectos de esta categoría, lo formativo tendrá relación con aspectos: formales, referidos a la formación académica de pregrado y postgrado, e informales, referido a lo aprendido desde las prácticas. Con respecto a los aspectos formales, desde los años 70, hasta mediados de los años 90, la formación académica es orientada desde enfoques y modelos biomédicos. En la formación de pregrado no estaban presentes como tales los conceptos de comunidad, sólo existían algunos elementos de lo comunitario enfocados a Salud Mental desde la institución.

“...Orientación a lo medico, clínico e institucional...”. (Entrevistado 2, generación del 77)

“...Era un trabajo mucho más individual y los grupos estaban en una sola asignatura, que era la socio terapia, .......que tienen que ver con el tema de los procesos intrahospitalarios, intrainstitucionales, nunca hacia la comunidad,...” (Entrevistado 3, generación del 87)

A mediados de los 90, se incorpora en las mallas curriculares la asignatura comunitaria, como un aspecto complementario hacia la intervención de la salud mental.

“...Había un ramo de comunitaria, que me marcó, pero no era con la conciencia de hoy en día, hoy se le dedica un año al ramo. La Orientación era biomédica, lo psicosocial relacionado con salud mental, lo comunitario era aceptado, pero primaba lo otro...”. (Entrevistado 6, generación del 98)

En las prácticas profesionales, se destaca la realizada en la ONG EPES (Educación Popular en Salud), estas prácticas fueron significativas en el desarrollo hacia una mirada comunitaria en la generación de los 90 .

“...Y al final de todo este transito vino el EPES y ahí otro cuento po', estaba todo el año esperando que llegara esa práctica quería que puro llegara, y fue distinto a lo que me entregó la universidad, que fue más bien desde la Terapia Ocupacional más biomédica, más mecanicista, y después, cuando empezó a ser comunitario fue otra cosa me dio un giro de 360 grados..." (Entrevistado 6, generación del 98)

En los años 2000, sigue siendo predominante la orientación biopsicosocial, pero existen elementos dentro de la misma formación que permiten ver diversas miradas, en estos años, aparecen como docentes Terapeutas Ocupacionales, en su mayoría, egresados en los años 80 que desarrollaron una mirada basada en su experiencia comunitaria.

“...No había un paradigma bien definido, había una separación entre Salud Mental y físico. Llegan profesores con este discurso, lo psicosocial y los diversos paradigmas y yo creo que por eso con un grupo de compañeros nos acercamos más a esta forma más crítica, y por eso te digo cuando llega A. G. y M. P., ellos llegaron con este concepto más instalado con esa propuesta de trabajo problematizadora...” (Entrevistado 10, generación del 2006) 
Por otro lado, se puede encontrar, que todos los Terapeutas Ocupacionales entrevistados, han complementado su formación, para contribuir y fundamentar las prácticas comunitarias con diversos programas de post título y postgrado como diplomados, magíster, entre otros.

“... yo no creo que mi práctica haya logrado consolidar un enfoque comunitario, el poder ponerle nombre yo creo fue un proceso posterior, y que ha sido más en parte del trabajo más académico, de mi formación en el magister, de poder ir poniéndole nombre a estos proceso y a estos debates..." (Entrevistado 8, generación del 95)

Con respecto a aspectos informales de la formación, los Terapeutas Ocupacionales entrevistados, de los períodos pre dictadura, dictadura y principios de la democracia, tuvieron una formación fuera del ámbito universitario. Por lo que las herramientas entregadas en la formación académica (técnicas grupales de intervención, ergoterapias y dinámicas grupales y creatividad), fueron utilizadas por Terapeutas Ocupacionales en ámbitos paralelos al universitario: en poblaciones, tomas de terreno, etc., donde trabajaban con la comunidad.

“...Yo empecé a trabajar un poquito antes de recibirme ah (...). Trabajaba más bien por razones política digamos, formando monitores de salud en un campamento en la zona oriente de Santiago, antes de terminar la carrera...” (Entrevistado 4, generación del 87)

“...Desde las ergoterapias, que las llamaban en esa época, el tema de lo sistémico, toda esta historia iba teniendo sentido, y era inevitable que yo lo fuera relacionando con mis vivencias comunitarias, con el tema de cómo generar oportunidades, generar significados o contenidos, desde lo cotidiano, lo de la vida diaria tiene que ver con la sencillez y la simpleza de posibilidades de hacer..." (Entrevistado 3, generación del 87)

Por lo tanto, lo comunitario en estas generaciones fue aprendido desde el hacer y posteriormente se encontró con la teoría. Como se refiere a continuación:

“...Tú empiezas haciendo esta pega y de repente te encuentras a un compadre que habla de lo mismo que estás haciendo tú, y le pone nombre. Y uno queda así como: "Ah, mira tú, me acabo de dar cuenta que soy experta en redes sociales”. Ni sabía yo que existían las redes sociales...” (Entrevistado 5, generación del 85)

Desde el año 1994, hasta la actualidad, se produce un fenómeno contrario a lo anteriormente descrito, en donde las asignaturas comunitarias son parte de las mallas curriculares de las universidades estatales y privadas, y por tanto las nuevas generaciones tendrían un desarrollo teórico más amplio de lo comunitario desde el pregrado.

“...En los exámenes de repente cuando vamos aterrizando las preguntas y les vamos diciendo, ya pero qué harías, ¡bueno pero yo conectaría las redes!, ¿pero qué?, ¿Cómo? a ver dame un ejemplo, pero no sé, pero para mí están evidente obvio en todo caso porque yo estoy en el otro lado, pero falta una noción práctica, porque además lo que yo creo que te decía también en las clases hayan las experiencias prácticas, ¿cómo se hace eso? en concreto, ¿quién lo ha hecho?, ¿que se ha hecho?, yo creo que la teoría sí, pero mira en mi vida personal la teoría yo la he integrado cuando he estado abierta a entenderla...” (Entrevistado 11, generación del 2002)

\section{Aspectos de la Motivación.}

Los Terapeutas Ocupacionales entrevistados, deciden estudiar la carrera por un elemento común que es el trabajo con personas. Este aspecto ha sido transversal en todos los períodos socio históricos. Sin embargo es el sentido dado al trabajo lo que diferencia a las distintas generaciones.

“...Yo sé que tuve una vocación social, siempre. Desde chico digamos, por mis padres, yo soy hijo de obreros, mis dos papás son obreros, sumado a esto tenía un vecino que me habla de carrera y en ese tiempo también visitaba la parroquia y ahí se enseñaba el modelo de sanación de Jesús. Lo que pasa es que a mí el sufrimiento de la gente me afecta mucho.” (Entrevistado 4, generación 87) 
“...Cuando salí del colegio yo sabía que me interesaba trabajar con personas, desde el ámbito de la salud y entonces lo que hice fue recorrer un hospital completo. Me pareció que la Terapia Ocupacional era una forma diferente de acercarse para trabajar con las personas..." (Entrevistado 9, generación del 95)

Se extrae con respecto a las motivaciones, el sentido social de los Terapeutas Ocupacionales hacia el trabajo comunitario. En los primeros dos períodos se centra claramente hacia el cambio social, en cambio, en el período post-dictadura, la orientación hacia el cambio social no es tan explícita e intencionada como en la generación anterior, siendo diversa en sus definiciones.

“...Necesidad de generar transformaciones sociales, buscar más justicia, Necesidad de generar comprensión de ese cambio social, generando las condiciones desde hoy...” (Entrevistado 4, generación del 87)

“...Tiene que ver con estar ahí, con la gente, con que ellos se cuestionen, cómo significan en la ocupación en los procesos en todo...”. (Entrevistado 10, generación del 2006)

Aparecen aspectos desfavorables en lo personal, hay costos que dicen relación con la situación de vida y las responsabilidades personales y familiares, en que el trabajo comunitario, tanto, por el compromiso humano que exige, como, por la retribución económica, no es posible.

“...Lo negativo, demasiados esfuerzos personales, de mucha vocación social, poco sueldo, trabajo en contextos de riesgo, desgaste personal. Replanteamiento de esas condiciones, ganas de no seguir así eternamente. Lo que da como resultado, que te vayas a otros lugares en donde tienes todo más seguro, te dan ganas de estabilidad, cuando tus prioridades cambian..." (Entrevistado 11, generación del 2002).

\section{Aspectos acerca de Las Prácticas Comunitarias.}

Los entrevistados de los períodos de pre dictadura y dictadura, definen el trabajo comunitario, como: participación, organización y la premisa de que las mismas comunidades son capaces de lograr cambios para mejorar su calidad de vida. Previo a la dictadura el contexto social promovía estas instancias de trabajo comunitario, en donde las personas participaban y se creía que los cambios sociales eran posibles. Al llegar la dictadura si bien hay un quiebre, la organización y participación de las comunidades continúan, aunque focalizadas en la lucha en contra del descontento que generaba el sistema represivo de dicho momento.

“...Nosotros estábamos más en una lucha contra Pinochet, contra un sistema, entonces para mí el trabajo comunitario es un trabajo político también, ¿cachai?, apuntaba a buscar más justicia, en materias súper especificas como, mejor alimentación para los niños, mas salud mental para las mujeres, etc...." (Entrevistado 4, generación del 85)

Desde la generación de los años 90 y 2000, se aprecia que se aborda lo comunitario desde el estado a través de las políticas públicas, lo que se define como intervención comunitaria, estas intervenciones, en su mayoría, van dirigidas hacia el sujeto individual en su relación con la comunidad y no la comunidad como sujeto de intervención.

“...Lo bueno es que nos dimos cuenta de los errores, que debimos haber trabajado con toda la comunidad, no sólo con los aliados. Sólo vimos el sujeto y sus relaciones, no a la comunidad entera y los actores como los vecinos que no estaban de acuerdo, la televisión, el equipo, etc. Este aspecto es fundamental, porque si no logras ver todos los sistemas caes en esto y tu intervención puede ser más perjudicial que favorable..." (Entrevistado 6, generación del 98) 
La institucionalización del trabajo comunitario, comienza a cuestionarse desde las generaciones de los 90. Si bien los Terapeutas Ocupacionales que se forman en las generaciones previas también viven este proceso, se desarrolla en mayor medida con la llegada de la democracia. Desde los terapeutas entrevistados que realizan su práctica en el período post-dictatorial surgen problematizaciones acerca de si es posible realizar trabajo comunitario desde los dispositivos de salud, educación, justicia, entre otros, se reconocen dificultades en implementar la intervención comunitaria. Aparece la idea de "área chica”o “interfase”, espacio que permite minimizar las barreras impuestas desde el modelo de trabajo institucional para realizar trabajo comunitario. El uso de este espacio es una manera de contrarrestar lo que está determinado desde la institución, pero el énfasis está puesto en cómo se ocupa este espacio desde el hacer.

"...Pienso que se puede hacer dentro y afuera. Puedo trabajar desde un consultorio, pero pertenezco también a esa comunidad o puedo ser un dirigente social y también hacer trabajo comunitario... y...si bien te dicen que realices cosas, dentro de tu espacio puedes actuar desde un enfoque comunitario o desde otra manera. Depende de lo que quieras lograr y como veas a la comunidad y la problematización de tus prácticas...” (Entrevistado 6, generación del 98)

Las prácticas comunitarias, dentro de los dos primeros períodos y principios del tercero, solían ser procesos más lentos y de mayor duración, los procesos se vivían, con la comunidad y no era extraño, ver equipos trabajando por años dentro de las poblaciones. En el tercer período hasta la actualidad, los procesos de intervenciones comunitarias son más cortos, debido a que se trabaja en relación a proyectos, que tienen un tiempo determinado de duración, lo que aspectos como los vínculos, el conocimiento de la comunidad y sus procesos, no logran desarrollarse completamente.

...."Nosotros teníamos una relación de largo plazo con ellos, para nosotros, o sea había una historia previa, de EPES, en un acuerdo de trabajo con una familia, entonces nosotros fuimos creando vínculos, porque lo primero que haces con la comunidad, lo primero es crear vínculos, ósea que la gente conozca, tú conoces la gente, establecer espacios de confianza, establecer un escuchar al otro para saber qué es lo que están buscando, qué es lo que quieren hacer"(entrevistado 4, generación del 87)

Las prácticas de los Terapeutas Ocupacionales del periodo dictadura, tienen relación con el fortalecimiento de las organizaciones, facilitar procesos de participación, reconstrucción del tejido social. La importancia de los espacios propios y de lo cotidiano dentro de los períodos previos a la dictadura y dictadura, resultan más evidentes, ya que el trabajo comunitario se realiza desde la comunidad.

“...Me fui dando cuenta, que la única profesional de todos los que estábamos ahí que tenía la capacidad de ver a las personas desde una multiplicidad de aspectos a la vez, era yo. Los otros profesionales no están formados para la cotidianeidad con el paciente o con la persona con la que estás trabajando, nosotros sí, nosotros vemos al paciente nuestro todos los días, tres veces a la semana, todo lo que se pueda...”. (Entrevistado 5, generación del 85)

En el primer y segundo período, la participación constituye un aspecto fundamental del trabajo, de manera de restablecer las instancias de participación pérdidas con la llegada de la dictadura, estableciéndose el trabajo popular como una forma de hacer consciente lo que estaba ocurriendo y favorecer la organización comunitaria En el periodo post dictadura, se problematiza el concepto de participación, distinguiendo entre la participación funcional, donde la persona participa limitada y sujeta al control social, y el concepto de participación real, en que hay una plena conciencia de participación. Se trabaja con la comunidad con enfoques que responden a la generación de sujetos pasivos y funcionales al sistema, generando limitantes en la participación de los mismos actores sociales frente a esta realidad. 
“...Me da más miedo este tipo de participación, ya que lo que genera son ciudadanos pasivos, sin molestias, en donde se cree que se participa pero no. Es necesario instancias de participación real, en donde se le enseñe a las personas a participar..." (Extracto del grupo de discusión, generación del 95).

“... uno pasa a ser agente de control social, entonces cuando uno rompe esa brecha y te das cuenta que estás haciendo eso y dejas de hacerlo tiene que romper el vínculo con el programa con el proyecto, porque no hay conversación posible y eso es súper difícil resolver, es un tema complejo porque no son compatibles...” (Entrevistado 3, generación del 87).

Como se ve en los resultados, las prácticas comunitarias son diversas, distinguiendose las intervenciones comunitarias ligadas hacia las políticas públicas, su determinación previa del hacer y su marco desde la institucionalidad y el trabajo comunitario, el cual tiene una orientación liberadora que favorece la participación y el fortalecimiento de las comunidades. Además, se revisan conceptos relacionados con la cotidianeidad y la participación, lo cual las autoras creen aportana la dimensión política del trabajo comunitario, aspectos que serán profundizados en el análisis y discusión.

\section{ANÁLISIS Y DISCUSIÓN}

Desde el eje de análisis Contexto Socio Histórico se discutirá en relación a dos de las categorías que emergen de los resultados: Aspectos Formativos y, Prácticas Comunitarias

Los resultados obtenidos, muestran que los contextos sociohistóricos determinan las formas de hacer prácticas comunitarias en Chile, y éstas, a su vez, influyen en la interacción con la comunidad favoreciendo o dificultando su participación social. El Terapeuta Ocupacional es parte de ese contexto sociohistórico por lo que su identidad, equipo de trabajo y su práctica comunitaria están configurados y construidos en esa cotidianidad. Se desprende que los contextos históricos, políticos y sociales van determinando las formas de participación y de realizar trabajo comunitario de los Terapeutas Ocupacionales en los distintos períodos descritos, e influyendo en los aspectos formativos, tanto formales como informales (1).

En el período 70-73 (pre-dictadura)se genera un desarrollo comunitario y de participación social en el país, es un período caracterizado por los cambios sociales en el país, en donde el Estado cumple un rol Protector, el cual se caracteriza por favorecer y asegurar la calidad de vida a los ciudadanos. También pueden reconocerse significativos avances en la participación sociopolítica y gremial de los sectores populares y trabajadores, lo que se ve detenido abruptamente y de manera determinante con la llegada de la Dictadura en el año 1973.

El segundo período socio histórico estudiado es la dictadura, en donde la represión política prohibió la organización de las personas, terminándose con el desarrollo de las prácticas comunitarias existentes. Se evidenciaron cambios tanto en lo económico, como en el ámbito de las políticas públicas, primó el asistencialismo y se anuló toda forma de participación social, lo que pasado algunos años acrecentó el descontento de la población. Las personas que por miedo dejaron de participar, vuelven desde opciones políticas y sociales, con un potente y evidente descontento frente a lo que ocurría. A partir de lo anteriormente dicho, el trabajo comunitario se realizó de manera clandestina en conjunto con otras personas e instituciones que en ese minuto abrieron espacios de participación, como la iglesia y las ONG's, algunos Terapeutas Ocupacionales comienzan a motivarse por esta línea de trabajo, dado que era impensado visibilizar el trabajo poblacional en medio de la represión presente. (1) 
Las autoras de la investigación extraen de los relatos, que los Terapeutas Ocupacionales no sólo realizaban análisis críticos y problematizadores acerca de la realidad política y social existente en Chile, sino que además actuaban desde el hacer, desde la práctica, como lo refiere Klandermas “protestar es transformar un descontento” (Montero 2006) (14)

En el periodo post-dictadura, con el advenimiento de la democracia, las formas de participación de las organizaciones se comienzan a desarticular. Las personas que realizaban trabajo comunitario en el período de la dictadura, vieron como de a poco este impulso se diluía, por cuanto las problemáticas psicosociales fueron abarcadas por el Estado. Chile a los ojos del mundo, fue visto como un país próspero, en vías de desarrollo, con capacidad de resolver sus problemas. Con esto, disminuye el aporte económico internacional, que eran unos de los principales soportes de las ONG's en Chile, resultando en que muchas de estas organizaciones desaparezcan. El Estado toma un rol activo en lo comunitario y lo psicosocial, institucionalizando el trabajo comunitario y definiendo desde las políticas públicas las necesidades de las comunidades. El trabajo comunitario, se hace desde un protocolo establecido, actuando como agente de control social, donde; si bien hay posibilidades de participación, existe un límite, donde es otro quien decide hasta donde llega la participación.

Por lo señalado anteriormente, los tres períodos históricos ya descritos evidencian los cambios y formas en que se han ido determinando formas del quehacer comunitario y la comprensión de los procesos no son aislados y que estarán presentes de manera transversal en las categorías que se presentarán a continuación.

\section{Categoría I: Influencia de los aspectos formativos en las prácticas comunitarias}

En los dos primeros períodos sociohistóricos destaca la formación informal la cual se desarrolla en un contexto que favorecía el sentido de comunidad, las personas evidenciaban como parte de su cotidianeidad la participación comunitaria. Al llegar la dictadura, lo informal se muestra como una alternativa de participación y de lucha frente al escenario político existente. En la actualidad el desarrollo de la formación formal prima sobre el aprendizaje informal.

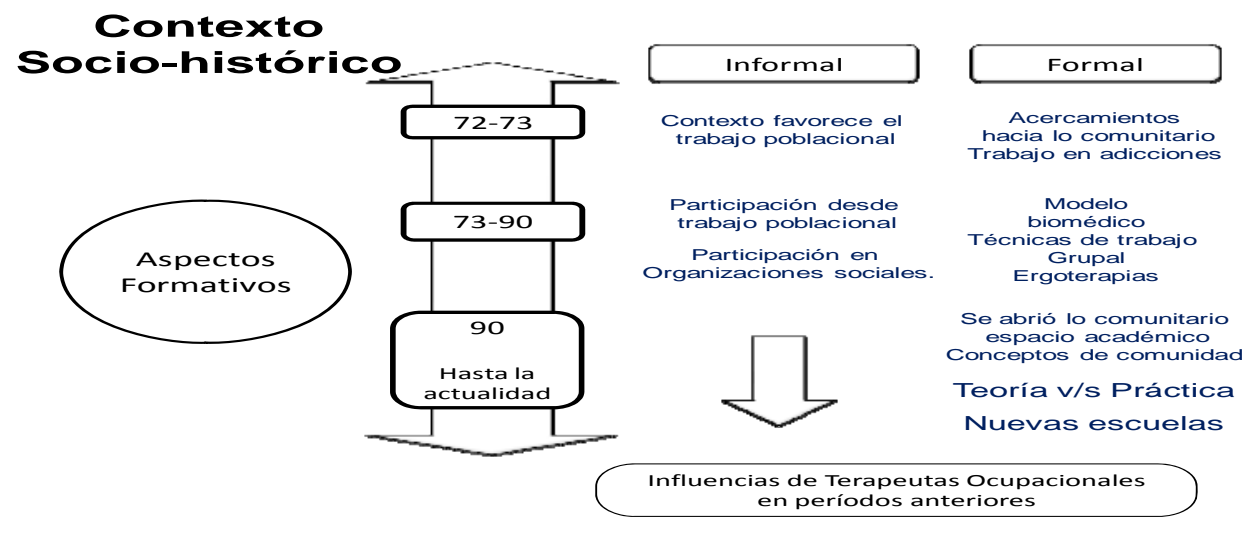

Figura I: Influencia de los aspectos formativos en las prácticas comunitarias

En el periodo de predictadura existía una formación formal médica tradicional y una experiencia práctica formativa informal en el trabajo poblacional de la época. Al iniciar la dictadura, se quiebra el trabajo poblacional. La Terapia Ocupacional, se inclina hacía modelos 
biomédicos, en que existía una clara tendencia, ligada a lo funcional e individual, y en actividades denominadas propias de Terapia Ocupacional. En esta generación existieron algunos estudiantes que manifestaban una postura crítica frente a la realidad académica, política y social, tomando el aprendizaje de la formación de Terapia Ocupacional como herramientas para llevarlas a cabo en otros contextos que tenían relación con la participación en organizaciones sociales para ayudar a la comunidad. Esto llevó a que los Terapeutas Ocupacionales de esta generación, comenzaran su desarrollo en lo comunitario desde su participación con la comunidad y posteriormente complementaron su formación con elementos teóricos.

Con la llegada de la democracia, en el año 94, en la escuela de Terapia Ocupacional de la Universidad de Chile, se incorpora la asignatura de Terapia Ocupacional comunitaria, abriendo un espacio en lo académico hacia el trabajo comunitario, en el reconocimiento al sujeto y su relación con el contexto.

En este período se comienzan a incorporar en las prácticas profesionales, centros con una mirada orientada hacía lo psicosocial y aparecen los primeros COSAM como centros de práctica y la ONG EPES (Educación Popular En Salud). En la generación de los 90, EPES fue influyente en el desarrollo de una mirada crítica y motivadora para realizar trabajo comunitario, abrió un nuevo enfoque de trabajo con las personas.

Dentro de los actores del trabajo comunitario, se destaca que los Terapeutas Ocupacionales que se formaron en los 70 y los 80 son los que influencian a las nuevas generaciones de Terapeutas Ocupacionales, desde sus prácticas y desde lo académico. Se podría decir entonces, que lo académico ha incorporado las experiencias de los Terapeutas Ocupaciones que han ido conceptualizando su práctica, pero que aún esta conceptualización no está suficientemente desarrollada desde los aportes teóricos propios de Terapia Ocupacional.

Los Terapeutas Ocupacionales formados en la actualidad, han tenido un desarrollo teórico acerca de lo comunitario, que ha facilitado y potenciado la problematización. Sin embargo, existe una brecha entre estos aspectos teóricos y su desarrollo práctico. Las autoras creen que se tendría que potenciar actividades prácticas desde la formación, ya que, a excepción del período de los 80, la participación en actividades sociales de los Terapeutas Ocupacionales de las últimas generaciones no es tan activa como la de los 80 .

\section{Categoría II Prácticas comunitarias de la Terapia Ocupacional}

Se encontró que no hay una definición única de prácticas comunitarias, pero sí se plantean distinciones, según los resultados, las autoras proponen que existen tres elementos centrales en toda práctica comunitaria los cuales son: la cotidianeidad, la experiencia y el significado que se le asignan a éstas, por lo cual, dependiendo del enfoque y modelo de trabajo es como estás se replicarán en la comunidad. Se distinguen dos grandes subcategorías en las prácticas comunitarias: el trabajo comunitario que fue posible de realizar en los dos primeros periodos sociohistóricos, debido a los factores contextuales que determinaron el hacer y la intervención comunitaria que es la que se realizaría en la actualidad, en donde el trabajo es desde la institucionalidad. 


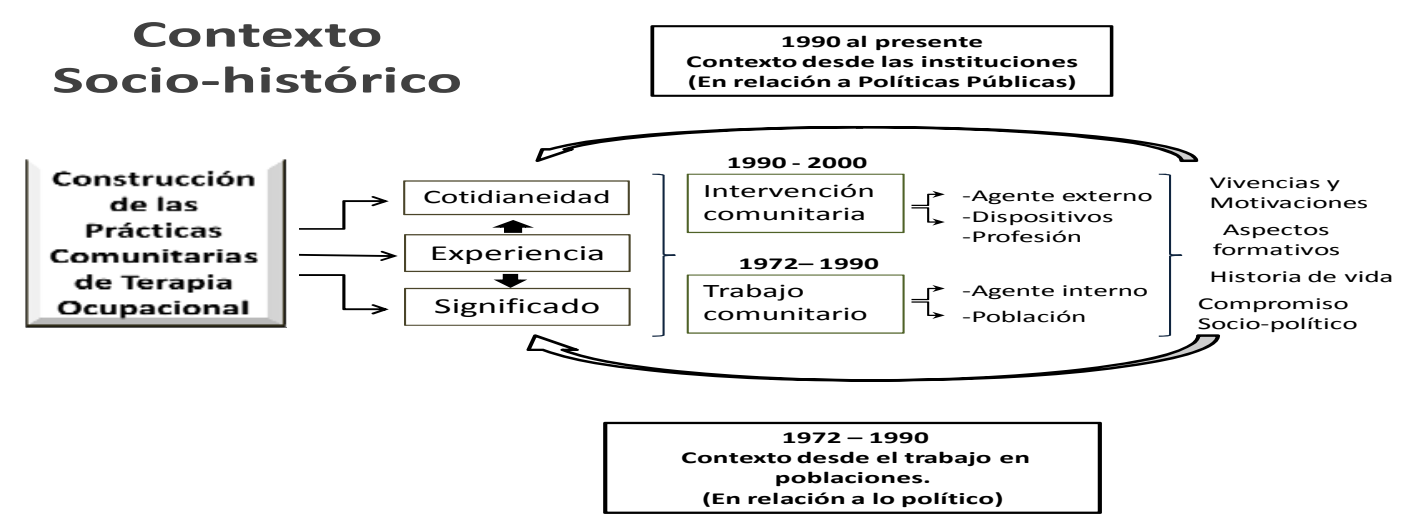

Figura II: Construcción de las prácticas comunitarias en los contextos socio históricas

Uno de los aspectos importantes es el perfil en común de los Terapeutas Ocupacionales que realizan trabajo comunitario, los cuales creen que las personas son gestores de sus propios cambios, se vinculan con las personas y las comunidades se evidencia un compromiso personal importante, con los afectos y con los tiempos.

Sin embargo, luego de realizar por mucho tiempo el trabajo comunitario se produce un cansancio, ganas de trabajar en condiciones menos adversas, lo que ha hecho que las generaciones de los 70 y 80 viven un proceso que ellos definen como un cambio de escenario en el trabajo comunitario, si bien ya no están en el terreno, algunos están en docencia, con lo cual traspasan sus experiencias de generación en generación y otros en las políticas públicas. Los Terapeutas Ocupacionales de los períodos post-dictatoriales, han decidido en base a sus orientaciones, algunos se dirigieron a las políticas públicas directamente, otros hacia las instituciones o directamente con las comunidades.

La política pública, como se dijo anteriormente, encuadra las intervenciones y enumera las prestaciones, pero hay un espacio desde la práctica del Terapeuta Ocupacional en donde se puede decidir si realiza la intervención tal como se la plantean o decide hacer cambios. Esa decisión, tan trascendental en el ámbito de lo comunitario, se cruza y se determina con las experiencias vitales en las prácticas. Es aquí en donde se hace una diferencia entre una intervención comunitaria la cual está ligada más a los aspectos del trabajo a lo profesional y el trabajo comunitario, que logran ver a la comunidad como el campo de las relaciones, en donde es fundamental las interacciones que se generan ahí.

Los Terapeutas Ocupacionales cuentan con la formación para aportar en el trabajo con la comunidad, sin embargo si éste no hace consciente sus prácticas y como éstas se han construido, pierde la posibilidad de generar cambio y por tanto, la dimensión política de su hacer. Ocuparse de la cotidianeidad en lo comunitario, implica una comprensión de la realidad sociohistórica, lo que determina las maneras de realizar las prácticas comunitarias de los Terapeutas Ocupacionales así como la construcción de comunidad.

\section{CONCLUSIONES}

El esfuerzo y la satisfacción que genera el realizar prácticas comunitarias ha sido fundamental para que las motivaciones de los Terapeutas Ocupacionales se sigan manteniendo o se vayan 
potenciado, de modo que se hace fundamental el traspaso de las experiencias no sólo desde el relato, sino tambien desde las vivencias que están estrechamente ligada a los significados, vínculos, afectos y compromiso con las comunidades. Esto va más allá de la relación desde la profesión, y es más bien una manera de mirar una realidad, situarse desde un sentido crítico, tanto desde lo aprendido como desde lo cotidiano, en que a todos estos aspectos se le atribuya un significado de carácter liberador hacia las personas, con el interés de movilizar a las personas hacia el cambio social, entendiendo que este cambio necesariamente se realiza en conjunto con otros.

Las investigadoras lograron apreciar como las experiencias comunitarias de los Terapeutas Ocupacionales del período de los de los 70 y 80, han sido traspasadas significativamente en el período de los 90 hasta la actualidad, y que si bien ha sido desde la formación académica, ha logrado dar sentido a quienes hoy tienen una mirada comunitaria.

Es importante considerar que no existe una sola forma de realizar las prácticas comunitarias, ya que una práctica comunitaria se puede realizar desde una intervención desde los dispositivos, o directamente en el trabajo comunitario en la comunidad a partir de organizaciones sociales u ONG's. Lo que se rescata del presente estudio, es que a pesar de que existan limitantes en términos institucionales o de políticas públicas, actuar a través del vínculo, y participar de instancias cotidianas con las personas hace posible que se pueda realizar una práctica comunitaria, por lo que las formas de actuar son diversas.

Para las autoras el compartir las distintas historias y experiencias transmitidas, a través los Terapeutas Ocupacionales de los diferentes períodos, permite reflexionar, acerca de las miradas y desafíos de la Terapia Ocupacional hacia el trabajo comunitario como campo de acción, en diferentes ámbitos del hacer. De tal modo que, a pesar de los fracasos y frustraciones, es tremendamente enriquecedor evidenciar que lo procesos de construcción, mantienen viva la fuerza hacia el fortalecimiento de las comunidades.

Es por esto que es necesario, el hacer consiente, el tipo de perspectiva con lo cual los profesionales se forman y como se va orientando la enseñanza recibida. Es vital estar siempre problematizando las propias prácticas con el fin de hacer consiente las diversas maneras de hacer trabajo comunitario.

Si bien existen muchos elementos en que hay que seguir problematizado e investigando, en relación a esto se plantean las siguientes interrogantes: ¿Cómo contribuir a los espacios problematizadores?, ¿Cómo poder hacerle frente a paradigmas que cada vez se vuelven más dominantes?, ¿La Terapia Ocupacional y los Terapeutas Ocupacionales están preparados, para mirar desde una conciencia crítica su trabajo?

Se espera que en un futuro, lo realizado en este seminario, sea sólo el inicio de un largo proceso de investigación en diversos aspectos y ámbitos tanto de la Terapia Ocupacional, como del trabajo comunitario, en donde se considere la importancia de las experiencias prácticas de las mismas personas que realizan trabajo comunitario. 


\section{REFERENCIAS BIBLIOGRAFICAS}

1 MONTERO M, Hacer para Transformar, El método de la Psicología Comunitaria, Argentina: Editorial PAIDOS, 2006

2 PESET J.L. Política y educación en la correspondencia familiar de Philippe Pinel, centro de estudios históricos C.S.I.C, 1991, 14, 589-602. Dialnet. Disponible en:

www.dialnet unirioja.es/servlet/fichero_articulo?codigo=62087. (consultado 25 de octubre 2008)

${ }^{3}$ CORTINES A., CONTRERAS F., CORTÉS G. Seminario de titulo, "Estudio retrospectivo de los Terapeutas Ocupacionales titulados de la Universidad de Chile entre 1966 y 1976”, Visiones y aportes de las 10 primeras promociones, Universidad de Playa Ancha 2005. Pág. 19

${ }^{4}$ WILLARD \& SPACKMAN, Terapia Ocupacional. EEUU: Editorial PANAMERICANA 2005.

5 KRONENBERG, ALGADO, SIMÓ, POLLARD. Terapia Ocupacional sin fronteras, Aprendiendo del espíritu de supervivientes. Editorial PANAMERICANA, 2007.

${ }^{6}$ MOLINAS DE RONDINA, J, , Aportes de la Terapia Ocupacional desde un modelo Constructivista, 2006

${ }^{7}$ CHAPARRO E.; FUENTES, J; MORÁN G., Diana; et al. La Terapia Ocupacional en la intervención en salud comunitaria: el modelo educativo. Revista Chilena de Terapia Ocupacional 2005; 5. Disponible en : www.revistaterapiaocupacional.cl (consultado 15 de noviembre de 2008)

${ }^{8}$ CELLA A y POLINELLI S.; Terapeutas Ocupacionales, Nuevos desafíos de Terapia Ocupacional Comunitaria. Revista chilena de Terapia Ocupacional 2006. Disponible en: www.revistaterapiaocupacional.cl (consultado 16 de noviembre de 2008

9 EQUIPO EPES, Temas de trabajo. Pagina web de EPES. Disponible en: $\underline{w w w . e p e s . c l}$ (consultado 20 de octubre 2008)

10 TEYLOR, S.J; BOGDAN, R., Introducción a los Métodos Cualitativos de Investigación, Editorial PAIDOS, 1987.

${ }^{11}$ MONTERO M., Introducción a la Psicología Comunitaria, Desarrollo, Conceptos y Procesos, Argentina: Editorial PAIDOS, 2004 